\title{
Factors contributing to sleep deprivation in a multi- disciplinary intensive care unit in South Africa
}

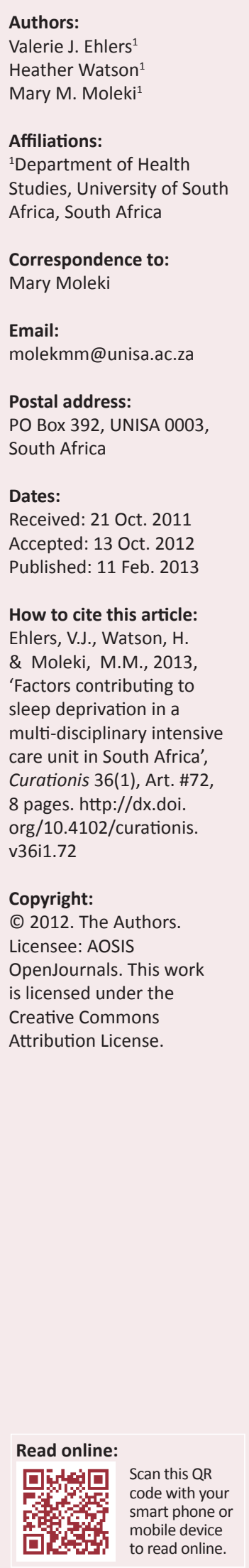

Patients in intensive care units require rest and sleep to recuperate, but might suffer from sleep deprivation due to ongoing unit activities. The study aimed to identify and describe the factors contributing to sleep deprivation in one multi-disciplinary intensive care unit (MDICU) in a private hospital in South Africa. Quantitative, descriptive research was conducted to identify factors contributing to sleep deprivation in the research setting, and to make recommendations to enhance these patients' abilities to sleep. Structured interviews were conducted with 34 adult non-ventilated patients who had spent at least one night in the MDICU and who gave informed consent. Out of the 34 interviewed patients $70.6 \%$ $(n=24)$ indicated that they suffered from sleep deprivation in the MDICU. The five major factors contributing to sleep deprivation in a MDICU were, (1) not knowing nurses' names, noise caused by alarms, (2) stress, (3) inability to understand medical terms, and (3) blood pressure cuffs that restricted patients' movements and smelled badly. Patients' abilities to sleep were enhanced by reassuring nurses whose names they knew and with whom they could communicate. By attending to the identified five major factors, patients' abilities to sleep in a MDICU could be enhanced enabling patients to recuperate faster. The implementation of such measures need not incur financial costs for the MDICU concerned.

\section{Introduction}

To suffer from a bad night's sleep every once in a whilst, is not an unusual occurrence. Human existence without any sleep at all, is unknown. Maslow, in his Hierarchy of Needs Model, refers to sleep (a state of natural rest), as a basic physiological human need. Sleep is an essential part of the 24-hour cycle, its purpose being to 'prevent physiological and psychological exhaustion' (Sleep and noise in the ICU 2008:1). Adequate rest and proper immune functioning are closely related. Sleep deprivation disturbs one's thinking patterns and weakens the body, making it more susceptible to disease (Cardinal 2006:12). The consequences of sleep deprivation include (1) physical effects, (2) mental impairment, (3) and mental health complications. Inadequate rest impairs one's ability to think, to handle stress, to maintain a healthy immune system, and to control emotions (Sleep Deprivation 2006). These effects are compounded for patients in intensive care units (ICUs) who require sleep to recuperate. Adequate rest and sleep should increase the likelihood and speed of recovery, shorten hospital stays and reduce medical expenses (Urden, Stacey \& Lough 2002:77).

Intensive care unit (ICU) environments are complex, stressful units (Rodriguez 2005:35). ICUs represent hostile environments for patients experiencing discomfort, pain, maintaining forced positions, coping with physical constraints, enduring nursing and medical procedures, noise, flashing lights and sleep disrupted by ICU activities. Noise has been described as a (Mazer 2005:9) 'primary cause of sleep deprivation and disturbance amongst patients'. Patients might hear conversations between staff members, visitors and patients, sounds of other patients in distress and mechanical devices, noises, nurses walking about, monitors' sounds, televisions and radios. Mazer (2005:9) further indicated that: '... patients exposed to continuous extraneous noise can also experience altered memory, increased agitation, less tolerance of pain, and feelings of isolation'. These experiences can impact negatively on ICU patients' recuperation processes.

The multi-disciplinary intensive care (MDICU) 24-hour routine, with many unfamiliar noises and activities, might make it impossible to distinguish day from night. In a windowless MDICU there is constant artificial light which removes the circadian rhythms' natural cues. In a MDICU, a fully conscious patient, who had a myocardial infarction, might lie next to a mechanically ventilated car accident survivor with multiple injuries, fractures and many intravenous treatments. Seriously ill patients requiring different types of critical interventions might contribute to patients' sleep deprivation levels in a MDICU.

'Sedation and analgesia ... involves the administration of drugs to produce a state of sedation and analgesia ... This medically controlled state of depressed consciousness allows the patient 
to: (1) maintain protective reflexes, (2) maintain an open airway independently and continuously, and (3) respond to physical stimulation or verbal command.' (Monahan, Sands, Neighbors, Marek \& Green 2007:244-2445). Sedation and analgesia might not be available to all ICU patients as their respiratory and cardiac functions might be affected by these drugs. Although sedation might enhance sleep for some patients, in others it might cause hallucinations, anxiety and aggravated sleeplessness. Sedation and analgesia are unlikely to counter balance factors contributing to patients' sleep deprivation in ICUs.

\section{Definitions of key concepts}

'Today's critical care unit continues to be a unique, highpaced environment in which the most sophisticated medical, nursing and technical interventions are integrated to combat life-threatening illness. These units are referred to as intensive care units (ICUs) ... and other names that identify the intensity of patient care. The one constant is that the role of nursing is critical to success.' (Monahan et al. 2007:192)

A multi-disciplinary intensive care unit provides intensive care to medical and surgical patients of both genders of all ages with any specialised needs.

Sleep refers to a state '... marked by reduced consciousness, reduced movement of the skeletal muscles, and slowed-down metabolism' (Sleep terms, definitions and abbreviations 2007).

Sleep deprivation refers to an acute or chronic lack of sufficient sleep. 'An essential part of the 24-hour cycle, sleep accounts for approximately one third of a person's life. An adequate amount of uninterrupted sleep is essential to prevent exhaustion or illness and maintain physiological and psychological well-being. Rapid eye movement (REM) sleep, important for mental restoration, occurs primarily in the last cycles of uninterrupted sleep and is the most likely form of sleep to be affected by sleep deprivation in the ICU ... recurrent disruptions in the sleep cycle quickly leads to a lack of REM sleep. Adverse effects of REM sleep deprivation include irritability, anxiety, physical exhaustion, disruption of metabolic functions, and respiratory distress' (Monahan et al. 2007:194).

\section{Problem statement}

Critically ill patients need to get enough sleep in the MDICU to enhance their recuperation processes. The problem was that patients continued to complain about sleeplessness in the participating MDICU.

\section{Purpose of objectives of the study}

The purpose of this study was to identify factors contributing to patients' sleep deprivation in one MDICU and to make recommendations to enhance these patient's abilities to sleep.
Research objectives, guiding this study, aimed to:

- identify factors influencing patients' abilities to sleep in one MDICU

- obtain patients' suggestions for enhancing their abilities to sleep in the MDICU

- make recommendations to address the factors contributing to patients' sleep

- deprivation in the participating MDICU.

\section{Research method and design}

A quantitative, descriptive design using structured interviews was adopted for this study. The 2nd author conducted structured interviews, determining how each patient experienced the previous night's sleep. No treatments or changes were introduced to any patient in order to collect data; structured interviews were conducted to determine how patients experienced their abilities to sleep, or not to do so, in the MDICU. Factors identified as contributing to sleep deprivation in the MDICU during data collection and analyses are described in quantitative terms.

\section{Population}

The research population comprised 99 patients who had spent at least one night in the participating MDICU during the time of data collection (November and December 2007). Patients comprising the accessible population had to be at least 21 years of age, not be ventilated, able to communicate in English, and willing to be interviewed after having spent at least one night in the MDICU concerned.

\section{Sample}

Out the 99 patients admitted to the MDICU during the data collection period, 34 (34.3\%) comprised the convenient sample, because they met the inclusive criteria, provided informed consent and agreed to be interviewed by the second author.

\section{Research instrument}

A structured interview schedule was designed, based on a literature review identifying factors contributing to patients' sleep deprivation in an ICU. This instrument comprised four sections concerning biographic information; specific factors in the MDICU that could influence sleep deprivation; specific factors enhancing the patients' abilities to sleep; and ways in which factors contributing to sleep deprivation could be addressed in the MDICU from the patients' perspectives.

\section{Data analysis}

A statistician compiled a spreadsheet according to the nature of the questions in the structured interview schedule, facilitating data entry. Answers to open-ended questions were grouped and quantified and also entered into the spreadsheet. The statistician assisted with the data analysis and interpretations. The Statistical Package for the Social Sciences (SPSS version 11.5) was used to enter and analyse 
the data whilst the MS Excel computer program was used to generate tables and graphs to summarise and display the research findings.

\section{Ethical considerations}

Ethical clearance was granted by the Research and Ethics Committee of the Department of Health Studies, University of South Africa. Permission was requested from and granted by the management of the participating hospital and by the manager of the participating MDICU. Every person interviewed also gave his or her informed consent. No person was coerced to participate and every interviewee could withdraw his or her participation at any stage without suffering any effects whatsoever. The 2nd author, an experienced ICU nurse, conducted all the interviews and ensured that no risks were involved, and if any patient became exhausted during the interview, or suffered any untoward consequence, the interview would be stopped and effective interventions implemented (this never occurred). Any patient could refuse participation or could refuse to answer specific questions, without incurring any negative consequences whatsoever. Anonymity was guaranteed because no name was written on any structured interview and confidentiality was maintained. Only the researchers and the statistician had access to the completed interview schedules, which were kept under lock and key. These documents, and the data entered onto a computer protected with a secure password, would be destroyed after the acceptance of the research report. No remuneration was paid, though the recommendations of this study might enhance future patients' abilities to sleep in the MDICU.

\section{Reliability and validity of the instrument}

The research instrument was pre-tested on four patients, who had spent at least one night in the MDICU, were not ventilated, were at least 21 years old, signed informed consent and agreed to be interviewed. The information from these four interviews was excluded from the data analysis of the actual study.

No changes were required as these patients understood the questions. The structured interviews were completed within 30 minutes. No discrepancies were noted between the structured interviews' responses during the pre-test and during the actual data collection phase, indicating consistency and reliability of the research instrument.

Internal validity refers to the extent to which the information obtained through the structured interviews is a true reflection of reality of factors influencing sleep deprivation in a MDICU, and not the result of irrelevant or extraneous variables (Burns \& Grove 2005:215). Two nurse researchers and two experienced ICU nurses agreed that every item in the structured interview schedule pertained to factors that can influence patients' sleep deprivation in a MDICU. Thus both face and content validity were acceptable, according to the evaluations of these four consulted experts.
However, threats to external validity included that a small convenient non-random sample of 34 patients were interviewed from one MDICU, limiting the possibility of generalising the findings of this study to other MDICUs, or even to all patients admitted to the participating MDICU.

\section{Results}

The total number of respondents will be indicated by $N=$ 34 , whilst any subgroup of respondents will be indicated by $\mathrm{n}$. The biographic data of the respondents will be discussed followed by an analysis of the factors contributing to these patients' sleep deprivation in the MDICU, according to Maslow's Hierarchy of Needs (2007). The following section will present a discussion of the findings.

\section{Biographic data}

Of the interviewed patients, $29.4 \%(n=10)$ were aged $61-70$, $26.5 \%(n=9)$ were $51-60$ years old, implying that $19(55.9 \%)$ of the respondents were at least 51 years old, and the average age was 56.1 years. There were more female $(52.9 \% ; n=8)$ than male $(47.1 \% ; n=16)$ respondents, and $47.1 \%(n=16)$ were employed, $35.3 \%(n=12)$ were pensioners and $17.7 \%$ $(n=6)$ were housewives. Of the respondents, $47.0 \%(n=16)$ reportedly slept fairly well at home, $35.3 \%(n=12)$ slept very well, and $17.6 \%(n=6)$ experienced fragmented sleep patterns. Out of the 34 respondents, $70.6 \%(n=24)$ reported that they did not get adequate sleep since their admissions to the MDICU, and could also not rest during the day, to make up for their lack of sleep during the night.

Respondents were classified into medical, surgical and cardiac categories. Cardiac patients appeared to sleep less, but this difference was not statistically significant. Of the respondents, $29.4 \%(n=10)$ had cardiac conditions, with $50.0 \%(n=5)$ of them reported sleeping well the first night in MDICU as a result of medication. The remaining five (50.0\%) cardiac patients identified noise as the main reason for sleep disruptions, despite medication. Neither the number of patients in the MDICU, nor the patient's bed position influenced the patients' abilities to sleep.

\section{Factors influencing sleep deprivation in a MDICU}

The discussion about the factors contributing to MDICU patients' sleep deprivation will be presented according to Maslow's Hierarchy of Needs (Maslow's Holistic Dynamic Needs Hierarchy 2007:1). Numerous and diverse needs were identified, necessitating the listing of needs that were identified by at least $30.0 \%$ of the respondents (Table 1 ). However, relevant needs, mentioned by fewer than $30 \%$ of the respondents, will be mentioned in the discussion of the findings.

\section{Physiological needs}

In terms of Maslow's Hierarchy of Needs (2007:1), physiological needs refer to basic requirements for sustaining life with oxygen, food and fluids forming the foundational 
TABLE1: Factors influencing patients' sleep deprivation in a multi-disciplinary intensive care.

\begin{tabular}{|c|c|c|}
\hline Maslow's Hierarchy of Needs & $N$ & $\%$ \\
\hline \multicolumn{3}{|l|}{ Physiological Needs } \\
\hline Thirst & 27 & 79.4 \\
\hline Pain & 31 & 91.2 \\
\hline Urinary catheters cause discomfort & 21 & 61.8 \\
\hline Blood pressure cuffs are uncomfortable & 30 & 88.2 \\
\hline Monitors- restricting movement & 30 & 88.2 \\
\hline Lines or tubes restricting movement & 25 & 73.5 \\
\hline Abdominal discomfort & 20 & 58.8 \\
\hline Noise caused by alarms & 31 & 91.2 \\
\hline Noises from patients & 20 & 58.8 \\
\hline Ongoing activities in ICU & 17 & 50 \\
\hline Coughing & 15 & 44.1 \\
\hline Blood pressure cuffs smell badly & 30 & 88.2 \\
\hline Lights & 22 & 64.7 \\
\hline Feeling hot at night & 11 & 32.4 \\
\hline \multicolumn{3}{|l|}{ Safety and Security Needs } \\
\hline Stress & 31 & 91.2 \\
\hline Anxiety & 24 & 70.6 \\
\hline Worries about their families & 20 & 58.8 \\
\hline Time orientation & 29 & 85.3 \\
\hline Inability to distinguish day from night & 28 & 82.4 \\
\hline Space in MDICU & 17 & 50 \\
\hline Unfamiliar environment & 16 & 47.1 \\
\hline Importance of knowing nurses' names & 32 & 94.1 \\
\hline Importance of doctors' avoidance of medical jargon & 30 & 88.2 \\
\hline Friendly nurses on admission & 28 & 82.4 \\
\hline Unfamiliar nurses affect sleep adversely & 19 & 55.9 \\
\hline Overhearing nurses' discussions & 19 & 55.9 \\
\hline On admission- nurses introduce themselves & 15 & 44.1 \\
\hline On admission- given needed information & 13 & 38.2 \\
\hline $\begin{array}{l}\text { On admission - adequate information about monitors, } \\
\text { lines, drips, alarms and tubes }\end{array}$ & 13 & 38.2 \\
\hline Nurses who talk about patients & 12 & 35.3 \\
\hline \multicolumn{3}{|l|}{ Esteem Needs } \\
\hline Lack of privacy & 22 & 64.7 \\
\hline Urinary catheters: no pants & 21 & 61.8 \\
\hline Hospital attire & 18 & 52.9 \\
\hline Dislike using urinals and/or bedpans & 11 & 32.4 \\
\hline
\end{tabular}

$N$, Given as number.

needs. Other physiological needs include maintaining a regular body temperature, exercise, rest and sleep, avoiding pain and excreting body wastes.

\section{Thirst and hunger}

Of the respondents $79.4 \%(n=27)$ reported that thirst contributed to their sleep deprivation. A few respondents reported that nasogastric tubes $(5.9 \% ; n=2)$ and oxygen masks $(44.1 \% ; n=15)$ caused sore throats, thirst and pain. Honkus (2003:5) explained that oxygen dries the nasal passages and intensifies thirst. Anti-bacterial mouth washes $(5.9 \% ; n=2)$ reportedly aggravated some patients' thirst'. Hunger was a concern of only $17.7 \%(n=6)$ of the respondents. Two out of these six respondents were on 'nil per mouth' whilst the other four were hungry despite having eaten.

\section{Pain}

Out of the 34 patients, 31 (91.2\%) experienced pain during their first night in the MDICU. Out of these 31 respondents, $30(96.8 \%)$ reported pain relief as a result of pain medication.
One respondent reported no pain relief as the patient in the next bed was dying and family members were distressed. Although $91.2 \%(n=31)$ respondents identified pain as contributing to sleep deprivation in the MDICU, $64.7 \%(n=22)$ reported that anxiety aggravated their pain. Ferns (2007:43) mentioned the link between pain, anxiety, frustration and fear. Uncontrolled pain triggers physical and emotional stress responses, which might aggravate sleeplessness (Morton, Fontaine, Hudak \& Gallo 2005:46) despite the administration of analgesics.

\section{Bowel and bladder functions}

Of the respondents, $61.8 \%(n=21)$ had urinary catheters and $38.2 \%(n=13)$ reported that this discomfort disturbed their sleep. Continuous intravenous therapy $(5.9 \% ; n=2)$ resulted in frequent voiding of urine. Out of 16 male respondents, $50.0 \%(n=8)$ found it difficult to pass urine whilst lying in bed. Some respondents $(32.4 \% ; n=11)$ disliked using urinals or bedpans and postponed their use as long as possible, depriving them of some sleep.

Respondents $(5.9 \% ; n=2)$ with diarrhoea reported feeling undignified and embarrassed when they had to use bedpans in close proximity to other patients.

\section{Physical discomforts}

Most respondents $(88.2 \% ; n=30)$ indicated that the blood pressure cuffs caused discomfort and pain, disturbing their sleep. For one $(2.9 \%)$ respondent, the blood pressure cuff instilled a sense of security. Of the respondents, $26.5 \%(n=9)$ indicated that the MDICU beds were uncomfortable, causing backaches.

Of the respondents, $88.2 \%(n=30)$ identified monitor leads as restricting movement and becoming entangled with intravenous lines, other tubes and their arms. Alarms went off when monitor lines disconnected and their finger oxygen saturation probes lost signals, restricting patients' movements, and disturbing their sleep. Of the respondents, $11.8 \%(n=4)$ feared the ventilators and their sounds, and were worried about accidentally touching these machines, disrupting their functions. Similar ICU fears were also reported by Morton et al. (2005:37) claiming that these experiences could create 'undue anxiety and the potential for physiological instability in vulnerable patients.

As many as $73.5 \%(n=25)$ of the respondents reported that lines and tubes connected to their bodies disturbed their sleep. Of the respondents $58.8 \%(n=20)$ reported that abdominal discomfort disturbed their sleep, although only six $(17.7 \%)$ were post-operative patients.

\section{Noise}

Most respondents (82.4\%; $n=28)$ said alarms and 50.0\% $(n=17)$ said ongoing activities, such as suctioning, disturbed their sleep. Despite sedation, 50.0\% $(n=17)$ mentioned that noise from ongoing activities in MDICU aggravated their sleeplessness. Admissions during the night, deaths 
and sounds such as telephones also disrupted their sleep as did coughing $(44.1 \% ; n=15)$ and snoring $(23.5 \% ; n=8)$. Twenty (58.8\%) respondents identified different sounds as disrupting their sleep whilst two (5.9\%) noted that noisy, confused patients disturbed their sleep. However, 14.7\% $(n=5)$ reported that alarms were initially disturbing, but that they got used to these sounds. Only three $(8.8 \%)$ respondents indicated that nurses should respond faster to alarms. Whilst $85.3 \%(n=29)$ of the respondents were not disturbed by nurses' walking about the unit at night, and $14.7 \%(n=5)$ said some noise was expected, 20.6\% $(n=7)$ reported feeling comforted by the nurses' footsteps. Urden $e t$ al. (2002) mentioned that some patients perceived activities in an intensive care unit as contributing to their safety.

\section{Smells, temperature and lights in the MDICU}

Most respondents $(88.2 \% ; n=30)$ complained about the bad smells of the blood pressure cuffs, $29.4 \%(n=10)$ noted that other unpleasant smells (chemical agents, perfumes, diarrhoea and vomit) contributed to their sleep disturbances. Only one respondent reportedly slept with a light on at home, but $64.7 \%(n=22)$ identified the lights in MDICU to aggravate their sleep deprivation. Maintaining a comfortable temperature throughout the night could help promote patients abilities to sleep according to nine (26.5\%) patients. Eleven patients (32.4\%) woke up feeling hot, and four (11.8\%) reported feeling too cold.

\section{Safety and security needs}

'If the physiological needs are relatively well gratified, there then emerges a new set of needs, which we may categorise roughly as the safety needs' (Maslow's Holistic Dynamic Needs Hierarchy 2007:1). A person seeks safe situations, stability and protection, freedom from fear, anxiety and disorder (Green 2007:4).

\section{Stress, anxiety and worries}

As many as $91.2 \%(n=31)$ of the respondents reported that the ICU environment caused stress, because they were scared $(n=5)$, worried $(n=4)$, anxious $(n=4)$, and concerned about their conditions $(n=2)$. Stress is defined by Morton et al. (2005:175) as a situation that exists when an organism is faced with any stimulus that causes disequilibrium between psychological and physiological functioning.

Of the respondents, $70.6 \%(n=24)$ reported anxiety as a result of being unable to sleep, whilst $64.7 \%(n=22)$ experienced anxiety as a result of pain. Although not questioned about death and dying, $11.8 \%(n=4)$ of the respondents noted that death in the MDICU caused anxiety, contributing to their sleep deprivation. Urden et al. (2002:205) mentioned that the ICU could be a frightening environment for patients.

Only $29.4 \%(n=10)$ of the respondents worried about their work and $58.8 \%(n=20)$, about their families disturbing their sleep. According to Morton et al. (2005:114), patients might worry about their diagnoses, prognoses, treatment, finances, loneliness and/or disturbing dreams.

\section{Time orientation}

All 34 (100\%) respondents could see a clock from their beds, and $85.3 \%(n=29)$ indicated that it helped them to sleep as it assisted them to keep track of time. However, a few respondents $(11.8 ; n=4)$ indicated that watching the clock was stressful. Not knowing whether it was day or night was disruptive as $82.4 \%(n=28)$ of respondents reported missing the sun. Having windows in the unit, would make it easier to keep track of time for $73.5 \%(n=25)$ of the respondents. Some respondents $(26.5 \% ; n=9)$ reported that whilst seriously ill, they were not concerned about windows, only when they felt better.

\section{Physical space}

Of the respondents, $17(50.0 \%)$ considered the space between the beds in the MDICU to be inadequate. Seven (20.6\%) respondents noted that doctors and nurses worked around trolleys, curtains, beds and other equipment due to lack of space. The unfamiliar environment of the MDICU aggravated $16(47.1 \%)$ patients' sleeplessness. Smeltzer and Bare (2008:116) mentioned that people tend to regard the space in their immediate vicinity as an extension of themselves, and any threatening person or situation in this space may lead to anxiety and discomfort.

\section{Communication challenges}

Almost all respondents $(94.1 \% ; n=32)$ mentioned that knowing the nurses' names, and doctors who avoided using medical jargon $(88.2 \% ; n=30)$ and used pictures to demonstrate the patients' problems $(88.2 \% ; n=30)$ helped to make them feel safe and secure. Of the respondents $82.4 \%(n=28)$ reported that nurses were friendly during their admissions but that unfamiliar nurses $(55.9 \% ; n=19)$ adversely affected their abilities to sleep as well as overhearing nurses' discussions (55.9\%; $n=19)$ and nurses' talking about patients (35.3\%; $n=12)$. However, $14.7 \%(n=5)$ reported that as long as the nurses were not loud, nurses' voices were comforting. Respondents appreciated it if nurses introduced themselves $(44.1 \% ; n=15)$ and provided the required information $(38.2 \%$; $n=13$ ) on admission.

A lack of caring by nurses was identified by $11.8 \%(n=4)$ respondents, but no specific reasons were given. Some respondents $(26.5 \% ; n=9)$ were unaware of the MDICU's phone's availability. All respondents knew that cell phones were disallowed in the MDICU, but $23.6 \%(n=8)$ mentioned that nurses and doctors used cell phones in the unit.

Of the respondents $8.8 \%(n=3)$ reportedly did not see their doctors often enough, and had insufficient discussions with their doctors. Three (8.8\%) patients found it disturbing if their doctors phoned without visiting them. Only three $(8.8 \%)$ patients complained about doctors who spoke to nurses and not to the patients.

\section{Esteem needs}

'All people in society ... have a need for a stable, firmly based, normally high evaluation of themselves, for self-respect or 
self-esteem, and for the esteem of others ... Satisfaction of the self-esteem needs leads to feelings of self-confidence, worth, strength, capability and adequacy, of being useful and necessary in the world. But thwarting of these needs produces feelings of inferiority, of weakness and of helplessness. (Maslow' Holistic Dynamic Needs Hierarchy 2007:1-2).

\section{Confidentiality, privacy and dignity}

Nurses' and doctors' discussions of patients' conditions at their bedsides, within hearing range of other patients and/or visitors, were reported by $26.5 \%(n=9)$ patients to be embarrassing as no confidentiality was maintained. However, $8.8 \%(n=3)$ of the respondents mentioned that when seriously ill, they were not concerned about this issue, only when they started recovering, they felt that their privacy had been invaded by such behaviour. This finding seems to support Maslow's assumption that once physiological needs have been met, the other higher order needs assume importance in a person's life.

Some patients' dignity was threatened by a reported lack of privacy during visiting hours $(14.7 \%$; $n=5)$, when conversations could be overheard by others. Some respondents $(14.7 \% ; n=5)$ stated that visitors might stare at patients. Curtains drawn between beds provided inadequate privacy $(11.8 \% ; n=4)$. One patient would have preferred visitors to come in groups, but only two visitors were allowed per bed during visiting hours. Morton et al. (2005:114) maintained that the presence of family members decreases a patient's vulnerability in an ICU and enhances his or her feelings of security. However, family members' presence in a MDICU could have negative consequences for other patients' privacy.

\section{Clothing and bed baths}

All $34(100 \%)$ respondents were physically independent before admission to the MDICU. Knowing that nurses were going to bed bath them in the morning did not disturb $79.4 \%(n=27)$ of the respondents' sleep. Two (5.9\%) female respondents $(n=2)$ would be reassured knowing that they would not be washed by male nurses.

Out of the 18 female respondents, $83.3 \%(n=15)$ stated that they would sleep better if they were allowed to wear their own pyjamas, whilst eight $(50.0 \%)$ of the 16 male respondents felt this way. Those $21(61.8 \%)$ respondents who had indwelling urinary catheters, and who could not wear pants, felt uncomfortable.

Two respondents $(5.9 \%)$ were upset because they were unable to cover their bodies and heads because the MDICU's monitors, tubes and hospital attire made this impossible.

\section{Self actualisation needs}

Once the physiological, safety, love and esteem needs have been satisfied, the self-actualisation need emerges striving to meet one's highest potential by using one's creative abilities and skills (Green 2007:7). No self-actualisation needs were verbalised or observed by the respondents, probably because their main level of focus was at the basic physiological (survival) level in terms of Maslow's Hierarchy of Human Needs.

\section{Transcendence needs}

These needs refer to a human being's desire to reach out to others and help them find self-fulfilment and realise their potential, whilst it also includes spiritual experiences which provide meaning to one's life (What are your basic needs? 2008:2). No needs at this level were verbalised.

\section{Discussion of the results}

Most respondents $(82.4 \% ; n=28)$ reported that on admission, MDICU nurses were friendly, displayed good attitudes and were approachable, and 91.2\% $(n=31)$ reported that nurses addressed visitors respectfully. However, in response to open-ended questions, some respondents $(20.6 \% ; n=7)$, indicated that nurses were 'just doing their jobs' and were not interested in their patients $(5.9 \% ; n=2)$, expected patients to know what is happening in a MDICU $(2.9 \% ; n=1)$, and were not helpful $(2.9 \% ; n=1)$. Lack of caring was noted by $11.8 \%$ $(n=4)$ of the respondents, without giving reasons for these comments. Most respondents $(88.2 \% ; n=30)$ mentioned that nurses gave adequate nursing care day and night, but $8.8 \%$ $(n=3)$ found nurses to be 'task orientated'. Of the respondents, $20.6 \%(n=7)$ were of the opinion that they could trust only some of the nurses. Of the respondents, $5.9 \%(n=2)$ reported that permanent MDICU nurses had to check on moonlighters. Most respondents $(88.2 \% ; n=30)$ preferred familiar nurses, and 19 (55.9\%) battled to sleep when unfamiliar nurses were allocated to them. With staff change-overs, eight (23.5\%) respondents had to establish new relationships with nurses which disturbed their sleep.

Most respondents $(94.1 \% ; n=32)$ reported sleeping better once they knew the nurses. The remaining 5.9\% $(n=2)$ reported that as long as the nurses seemed to be doing their job, it did not matter. If patients were feeling uncomfortable with a nurse around their bed, they would talk to the nurse $(38.2 \% ; n=13)$, whilst $38.2 \%(n=13)$ would keep quiet, remain powerless to effect change, and $11.8 \%(n=4)$ would talk to a familiar MDICU nurse.

When asked what could be done to prevent patients feeling uncomfortable with new nurses, $26.5 \%$; $(n=9)$ indicated that nurses should communicate; $38.2 \%$; $(n=13)$ said having a familiar nurse around eases discomfort; $11.8 \%$; $(n=4)$ felt that new nurses should introduce themselves; $20.6 \%$; $(n=7)$ linked the need for communication to their ability to relax; $5.9 \%$; $(n=2)$ linked communication with trust.

\section{Limitations of the study}

The conclusions need to be interpreted against the limitations of the study. The study was only conducted in one MDICU, consequently the research findings might not be generalised 
to other settings. Only 34 conveniently selected patients were interviewed, limiting the possibility of generalising the findings. Only patients who volunteered to participate in the study were interviewed. It cannot be assumed that those who were willing to participate had similar experiences to those who refused to participate. Maslow's Hierarchy of Human Needs proved not to be a meaningful framework for contextualising the study, because most patients' needs were only at the physiological (basic) needs level.

\section{Recommendations}

The recommendations are divided into two sections: measures that doctors and nurses can implement and physical measures that can help to enhance patients' abilities to sleep in a MDICU.

\section{Measures that doctors and nurses can take to enhance multi-disciplinary intensive care patients' abilities to sleep}

Doctors should visit patients regularly in the MDICU, inform patients about their condition in understandable lay terms, phone the MDICU between visits to enquire about the patients' well-being, and talk to the patient rather than to the nurse. There should be sufficient time for patients to raise their concerns during doctors' visits. Doctors and nurses should not use medical jargon, should not talk about patients within hearing distance of other patients, include patients in their conversations and ensure that patients understand their conditions and their treatments.

Nurses who work in the MDICU should be friendly, approachable and respectful at all times. Nurses should introduce themselves and ensure that every patient knows who to call for assistance.

All patients in the MDICU should be informed about the accessibility of the MDICU phone. Nurses and doctors should not use cell phones in the unit. Doctors and nurses should ensure that effective pain medication is administered to patients. Nurses should enhance patient's comfort by ensuring that patients are not thirsty or hungry (if possible), patients do not hesitate to ask for bedpans and/or urinals, patients are reassured about the tubes, intravenous fluids and monitors around their beds.

\section{Physical measures that can be taken to enhance patients abilities to sleep in the multi-disciplinary intensive care unit}

Noise must be controlled. The volumes of alarms, telephones and television sets should be subdued. Nurses should talk quietly. A clock, and preferably the sun through windows, should be visible to assist patients' time orientation. Lights should be dimmed at night.

Blood pressure recordings should only be done at the required preset intervals to reduce discomfort. Bad smelling blood pressure cuffs should be replaced. Where possible, patients should be allowed to wear their own night clothes; visiting hours should be more lenient; a dying person should be moved to a private ward to provide privacy to his or her family members and to reduce the stress and anxiety of the other patients in the MDICU.

\section{Conclusions}

The following factors had the most significant impact on patients' sleep deprivation: not knowing nurses' names $(94.1 \% ; n=32)$; noise caused by alarms $(91.2 \% ; n=31)$; stress $(91.2 \% ; n=31)$; inability to understand medical terms used by doctors $(88.2 \% ; n=30)$; blood pressure cuffs restricting movement and having bad smells $(88.2 \% ; n=30)$; restriction of movement by monitors $(88.2 \% ; n=30)$; alarms $(82.4 \%$; $n=28)$; friendly, approachable and respectful nurses when admitted to the MDICU $(82.4 \% ; n=28)$; time disorientation $((82.4 \% ; n=28)$; thirst $(79.4 \% ; n=27)$; restricted movement due to lines and tubes $(73.4 \% ; n=25)$.

Despite the best surgical techniques and drugs, critically ill patients require sleep to recuperate. Nurses and doctors can enhance MDICU patients' abilities to sleep, make their stay in the MDICU more pleasant, and assist them to regain their physical strength more rapidly, without increasing financial costs.

\section{Acknowledgements}

We wish to thank the participating hospital and MDICU for granting permission for data collection and the Research and Ethics Committee, Department of Health Studies, University of South Africa for granting ethical clearance for conducting the study. Every patient who agreed to be interviewed is thanked for his or her contribution, without which this research project could not have been completed.

\section{Competing interests}

No funds were received for this project and the participating hospital imposed no limits on the study, except for the request to remain anonymous.

\section{Authors' contributions}

H.W. (University of South Africa) conducted the study for her master's dissertation, M.M.M. (University of South Africa) was the supervisor and V.J.E. (University of South Africa) was the joint supervisor. H.W. obtained permission from the hospital and conducted the structured interviews. M.M.M. and a V.J.E. guided the research process from its concept phase till its conclusion. M.M.M. and a V.J.E. assumed the major responsibility for getting the article written and revised based on the Master's dissertation, with inputs from H.W.

\section{References}

Burns, N. \& Grove, S.K., 2005, The practice of nursing research: conduct critique, and utilization, W.B. Saunders, Philadelphia.

Cardinal, F., 2006, 'This is your brain without sleep', viewed 23 June 2006, from http:// sleepdisorders.about.com/cs/sleepdeprivation/a/brainsleep 3.htm

Drench, M.E., Noonan, A.C., Sharby, N. \& Ventura, S.H., 2003, Psychosocial aspects of health care, Prentice Hall, Upper Saddle River. 
Green, D.C., 2007, 'Classics in the history of psychology', viewed 10 June 2007, from http://psychclassics.yorku.ca/Maslo/motivation.htm .

Honkus, V.L., 2003, 'Sleep deprivation in critical care units', Critical Care Nursing Quarterly 26 (3), 179-191. PMid: 12930033

'Maslow's Holistic Dynamic Needs Hierarchy', 2007, viewed 16 April 2007, from http://pandc.ca/?cat=abraham_maslow\&page=needs_hierarchy

Mazer, S.E., 2005, 'Stop the noise. Reduce errors by creating a quieter hospital environment', viewed 30 August 2007, from http://www.psqh.com/marapros/ noise.html

Monahan, F.D., Sands, J.K., Neighbros, M., Marek, J.F. \& Green, C.J., 2007, Phipps' medical-surgical nursing: health and illness perspectives, Mosby/Elsevier, Philadelphia.

Morton, P.G., Fontaine, D.K., Hudak, C.M. \& Gallo, B.M., 2005, Critical care nursing. A holistic approach. 8th edn. Lippincott Williams \& Wilkins, Philadelphia.
Rodriguez, A.M., 2005, 'Psychosocial adaptation in relatives of critically injured patients admitted to an intensive care unit', The Spanish Journal of Psychology $8(1), 36-44$

'Sleep and noise in the ICU', 2008, viewed 23 May 2008, from http://www.i-canuk. co.uk/article. aspx?articled=100823.

'Sleep deprivation', 2006, as viewed 23 June 2006, from http://www.sleepdeprivation.com.html/effectsphp3

'Sleep terms, definitions and abbreviatons', 2007, viewed 10 June 2007, from http:// www.sleepnet.com/definition.html

Smeltzer, S.C. \& Bare, B., 2008, 'Brunner and Suddarths textbook of medical-surgical nursing', 10th edn., Lippincott Williams \& Wilkins, Philadelphia.

Urden, L.D., Stacy, K.M. \& Lough, M.E., 2006, 'Thelan's critical care nursing. Diagnosis and management', 4th edn., C.V. Mosby, St Louis. PMid:16766905

What are your basic needs? 2008, viewed 16 May 2008, from http://72.30.186.56/ search/cache?ei=UTF8\&p=maslow+hierarchy=critical=care\&vhs.avg\&u=ww 